\title{
Organic Photovoltaic Behaviour with Centimeter-long Lateral Junctions
}

\author{
Jaseela Palassery Ithikkal ${ }^{1,3}$, Adrien Girault ${ }^{1,2}$, Mitsuru Kikuchi ${ }^{1}$, Yusuke Yabara ${ }^{1}$, \\ Seiichiro Izawa ${ }^{1,3}$, Masahiro Hiramoto ${ }^{1,3^{*}}$ \\ ${ }^{1}$ National Institutes of Natural Sciences, Institute for Molecular Science, \\ 5-1 Higashiyama, Myodaiji, Okazaki, Aichi, 444-8787, Japan \\ ${ }^{2}$ École nationale supérieure de chimie de Paris, 11 Rue Pierre et Marie Curie, 75005 \\ Paris, France \\ ${ }^{3}$ The Graduate University for Advanced Studies (SOKENDAI), 5-1 Higashiyama, \\ Myodaiji, Okazaki, Aichi, 444-8787, Japan
}

\begin{abstract}
We report organic photovoltaic cells with centimeter-long lateral junctions reaching $1.8 \mathrm{~cm}$. These junctions were formed between organic semiconductor films with extremely high mobilities for holes and electrons. The lateral diffusion lengths of photogenerated electrons and holes reached 4.7 and $5.5 \mathrm{~mm}$, respectively. The operation of the centimeter-long lateral junctions was dominated by trap-assisted recombination between the electrons and holes.
\end{abstract}

Keywords: organic photovoltaic cell, centimeter-long lateral junction, extremely high mobility, trap-assisted recombination, bimolecular recombination.

\footnotetext{
*Author to whom correspondence should be addressed;

E-mail address: hiramoto@ims.ac.jp
} 
Recently, organic semiconductors with remarkably high carrier mobilities reaching $40 \mathrm{~cm}^{2} \mathrm{~V}^{-1} \mathrm{~s}^{-1}$ have been reported [1-4]. Such high mobility was observed for carriers moving laterally through the material because of $\pi-\pi$ stacking parallel to the organic crystal surface.

Collection and lateral transport of photogenerated electrons and holes can free us from the vertical transport of carriers; consequently, we are freed from the limitation of the vertical thickness of conventional organic photovoltaic cells with sandwich-type structure [5-8]. Based on these considerations, we studied lateral-type organic photovoltaic cells using high-mobility organic semiconductors, such as single crystals $[9,10]$ and crystalline films [11]. However, the potential and determining factors for achieving macroscopically long lateral junctions remain unclear. Therefore, in this study, we focused on the dependence of photovoltaic properties on the macroscopic lengths of lateral junctions. We adopted C8-BTBT [Fig. 1(a)] with a high hole mobility of 43 $\mathrm{cm}^{2} \mathrm{~V}^{-1} \mathrm{~s}^{-1}[12,13]$ and PTCDI-C8 [Fig. 1(a)] with a high electron mobility of 1.7 $\mathrm{cm}^{2} \mathrm{~V}^{-1} \mathrm{~s}^{-1}[14]$.

In this paper, the operation of centimeter-long organic lateral junctions reaching $1.8 \mathrm{~cm}$ is reported.

Fig. 1 shows the structure of a lateral junction cell. Unpurified C8-BTBT (Nippon Kayaku Co., Ltd.) and PTCDI-C8 (Luminescence Technology) were used as the donor and acceptor, respectively. Organic semiconductors were deposited at $10^{-6} \mathrm{~Pa}$ and room temperature in an oil-free vacuum chamber housed in a glove box (EpiTech Inc., 12ET12007). The evaporation rate of both C8-BTBT and PTCDI-C8 was $0.1 \mathrm{~nm}$ $\mathrm{s}^{-1}$. The thickness of the C8-BTBT and PTCDI-C8 films was $50 \mathrm{~nm}$. Using a customized movable-mask system (EpiTech Inc.) [Fig. S1(a)], the C8-BTBT and PTCDI-C8 films were connected to $100 \mathrm{~nm}$-thick Ag electrodes through a $10 \mathrm{~nm}$-thick 
$\mathrm{MoO}_{3}$ and $10 \mathrm{~nm}$-thick bathocuproine (BCP); $\mathrm{BCP} / \mathrm{Ag}$ and $\mathrm{MoO}_{3} / \mathrm{Ag}$ acted as the electron- and hole-collecting electrodes, respectively [Fig. S1(b)]. The distance $(L)$ between the electron-collecting electrode (BCP/Ag) and hole-collecting electrode $\left(\mathrm{MoO}_{3} / \mathrm{Ag}\right)$ was varied from 0.2 to $18 \mathrm{~mm}$. A photograph of the cell with $L=18 \mathrm{~mm}$ is shown in Fig. 1(b). Energetic diagram of C8-BTBT and PTCDI is shown in Fig. 1(c).

Current-voltage $(J-V)$ characteristics were measured under simulated solar light irradiation (AM1, $100 \mathrm{mWcm}^{-2}$; ASAHI Spectra, HAL-320) in $\mathrm{N}_{2}$ atmosphere. The intensity of simulated solar light was changed from 0.1 to 10 sun using a neutral density filter to measure the light intensity dependence. To determine the hole and electron diffusion lengths, the cell surface of the light-irradiated side was covered with a movable mask from the hole-collecting electrode to the electron-collecting electrode or vice versa [Fig. 4].

Fig. 2(a) shows the $J-V$ characteristics of the cells [Fig. 1(a)] with $L=0.2$ (green curve), 0.7 (black curve), 2 (blue curve), and $18 \mathrm{~mm}$ (red curve). The vertical axis corresponds to the short-circuit photocurrent density per cell area $\left[J_{\mathrm{sc}}\right.$, Fig. 1(a), red rectangle]. Remarkable photovoltaic characteristics were observed for all cells [Fig. 2(a)]. Surprisingly, even a lateral cell with an $L$ of $1.8 \mathrm{~cm}$ [Fig. 2(a), red curve] exhibited a clear photovoltaic behavior. The $J_{\mathrm{sc}}$ steeply decreased from $4(L=0.2 \mathrm{~mm})$ to $0.015 \mu \mathrm{Acm}^{-2}$ ( $L=18 \mathrm{~mm}$ ) [Fig. 2(b)]. The open-circuit photovoltage ( $\left.V_{\mathrm{oc}}\right)$ gradually decreased from $0.79(L=0.2 \mathrm{~mm})$ to $0.42 \mathrm{~V}(L=18 \mathrm{~mm})$ [Fig. 2(c)]. Moreover, the fill factor $(\mathrm{FF})$ gradually decreased from $0.56(L=0.2 \mathrm{~mm})$ to $0.32(L=18 \mathrm{~mm})$ [Fig. 2(d)]. Although the photocurrent was still small, photovoltaic behaviour of centimeter-long lateral organic junctions was observed.

To clarify the mechanism of photocarrier generation in the centimeter-long lateral junctions, the dependences of $J-V$ characteristics on light intensity from 0.1 to 10 sun were measured [Fig. 3(a)]. Photocurrent density increased with increasing the light 
intensity. Striking $J-V$ curves were observed even under 10 sun irradiation at $L=2 \mathrm{~mm}$ regardless of the appearance of resistive effect. At $L=2 \mathrm{~mm}$, a slope of 0.95 was observed from the relationship between $J_{\text {sc }}$ and light intensity [Fig. 3(b)]. Fig. 3(c) shows the dependence on $L$ of the slope of the light intensity plots in Fig. 3(b). At an extremely small $L(0.2 \mathrm{~mm})$, the slope approached unity $(0.99)$. When the $L$ was increased from 2 to $18 \mathrm{~mm}$, a slight decrease in the slope was observed. The slope reached 0.90 at $L=18 \mathrm{~mm}$. These results indicate the absence of bimolecular recombination at approximately $L=0.2 \mathrm{~mm}$ and the minimal effect of bimolecular recombination between the holes in the C8-BTBT film and the electrons in the PTCDI-C8 film [15] at $L=2 \mathrm{~mm}$ to $18 \mathrm{~mm}$. Minimal bimolecular recombination occurred in a lateral cell even for an extraordinary $L$ of $1.8 \mathrm{~cm}$.

Fig. 3(d) shows the semi-logarithmic plot between $V_{\text {oc }}$ and light intensity. Linear relationship was observed. The slope is expressed as $n k T[16,17,18]$. Here, $n, k$, and $T$ are the diode factor, Boltzmann constant, and absolute temperature, respectively. Therefore, we can determine $n$ from the slope. In ideal $p-n$ junctions, $n$ is equal to unity. An $n$ value greater than unity indicates the presence of trap-assisted recombination $[19,20]$. Thus, we concluded that minimal trap-assisted recombination existed at $L=0.2$ $\mathrm{mm}$, and trap-assisted recombination occurred significantly at $L=2-18 \mathrm{~mm}$ [Fig. 3(e)]. Although the value of $\mathrm{n}$ normally ranges from 1 to 2 , when the diode quality is very poor, $\mathrm{n}$ value larger than 2 was reported [21]. We think that $\mathrm{n}$ value even higher than 4 represents the very poor diode quality of lateral cells having large L, which often showed little rectification behaviour together with the dominance of trap-assisted recombination.

To analyze the effects of photogenerated electrons and holes on the lateral cell performance, movable-mask experiments were performed [Fig. 4]. For holes, the movable mask was inserted between the cell surface and irradiated light from the hole- 
to the electron-collecting electrode [Fig. 4, upper]. For electrons, the movable mask was inserted from electron- to hole-collecting electrode [Fig. 4, lower]. Fig. 4(middle) shows the semi-logarithmic plots between $J_{\mathrm{sc}}$ and the distance of mask edge from the hole-collecting electrode (blue dots) for cells with $L=4,6$, and $18 \mathrm{~mm}$. The $J_{\mathrm{sc}}$ gradually decreased as the mask was moved to the electron-collecting electrode. Moreover, the observed values of $\mathrm{J}_{\mathrm{sc}}$ drops exponentially with increasing distance. Here, we define the hole diffusion length $\left(L_{\mathrm{h}}\right)$ to the $\mathrm{L}$ value showing the photocurrent density of $(1 / \mathrm{e}) \mathbf{J}_{\mathrm{sc}}(\mathrm{L}=0 \mathrm{~mm})$ as shown in Fig. S3. The values of hole diffusion length $\left(L_{\mathrm{h}}\right)$ for $L=4,6$, and $18 \mathrm{~mm}$ were $5.3,6.3$, and $4.8 \mathrm{~mm}$, respectively; these values were determined from the slopes of the lines. By averaging these values, we obtained the $L_{\mathrm{h}}$ $(5.5 \mathrm{~mm})$. Furthermore, Fig. 4(middle) shows the semi-logarithmic plots between $J_{\mathrm{sc}}$ and the distance of mask edge from the electron-collecting electrode (red dots) for cells with $L=4,6$, and $18 \mathrm{~mm}$. The electron diffusion length $\left(L_{\mathrm{e}}\right)$ was $4.7 \mathrm{~mm}$, which was determined using the same analysis. The $L_{\mathrm{e}}(4.7 \mathrm{~mm})$ and $L_{\mathrm{h}}(5.5 \mathrm{~mm})$ values were macroscopic and extraordinarily long.

Fig. 5(a) shows the schematics of the hole movement with (lower) and without (upper) the movable mask. Without the movable mask, most holes photogenerated near the hole-collecting electrode were collected. With the movable mask (lower), most holes photogenerated away from the hole-collecting electrode were not collected; that is, the holes were captured by the traps. Hence, the movable-mask experiment revealed the $L_{\mathrm{e}}$ and $L_{\mathrm{h}}$, which were dominated by the trapping of electrons and holes, respectively. The trapping process is directly linked to the trap-assisted recombination as shown in Figs. 5(d) and 5(e).

Fig. 5(b) shows the profile of the portions of electrons (red curves) and holes (blue curves) that can be collected by the electron- and hole-collecting electrodes. Profiles drop exponentially with increasing the distance. The red and blue curves were 
plotted based on the $L_{\mathrm{e}}(4.7 \mathrm{~mm})$ and $L_{\mathrm{h}}(5.5 \mathrm{~mm})$, respectively. To generate photocurrent, both the photogenerated electrons and holes should be extracted by the respective electrodes. This mechanism indicates that only the photogenerated carriers in the green-shaded region appeared as the photocurrent. Fig. 5(c) shows the dependence of $J_{\mathrm{sc}}$ on $L$. The blue curve is the calculated $J_{\mathrm{sc}}$ from the area of green-shaded region. The red curve shows the observed $J_{\text {sc }}$. The considerable decrease in photocurrent was attributed to the trap-assisted recombination shown by the blue curve; this decrease was calculated from the $L_{\mathrm{e}}$ and $L_{\mathrm{h}}$ dominated by the traps (Fig. 5(a)). The calculated and observed $J_{\mathrm{sc}}$ should be equal in an extremely small $L(<0.4 \mathrm{~mm})$ region because of the absence of bimolecular recombination; hence, we could adjust the two curves in this region. A difference was observed between the calculated (blue) and observed (red) curves because of another recombination process for large $L$. We concluded that this process was the bimolecular recombination because the slope of the light-intensity dependence line [Fig. 3(c)] was significantly smaller than unity (0.90) for $L>2 \mathrm{~mm}$. At $L=18 \mathrm{~mm}$, the percentages of trap-assisted [Figs. 5(d) and 5(e)] and bimolecular [Fig. 5(f)] recombinations were $94 \%$ and $6 \%$, respectively. The increased trap-assisted recombination with increasing $\mathrm{L}$ can be reasonably explained based on the increase in the total number of hole and electron traps. If we assume that the traps are formed at molecular defects, such as molecular vacancy and molecular dislocation, we might further increase the lateral junction length by removing such defects.

In conclusion, photovoltaic behaviours of centimeter-order organic lateral junctions were observed. This was accomplished through the extremely long diffusions for holes $(5.5 \mathrm{~mm})$ and electrons $(4.7 \mathrm{~mm})$. In long lateral cells, trap-assisted recombination controlled the magnitude of photocurrent density. Though the magnitude of photocurrent is small in the present, we believe the advanced technologies for 
organic film fabrication would remove the defects acting as traps in the organic semiconductor films and would accomplish the significant increase of photocurrent. We think that extremely high series resistance can be overcome by using the doping technique to organic semiconductors [22]. Centimeter-long carrier diffusions would pave the way the lateral multilayer organic photovoltaic cells [11] which is feed from the route formation issue in the blended junction.

\section{Supplementary Material}

See supplementary material for the detailed design of customized movable mask system and the device structure of the lateral cell (Fig. S1), the method used for determining the diffusion length of carriers (Fig. S2).

\section{Acknowledgments}

We greatly appreciate the funding from JSPS KAKENHI (No. 17K19110). We appreciate the internship program between Chimie ParisTech and IMS.

\section{Data Availability}

The data that support the findings of this study are available from the corresponding author upon reasonable request. 


\section{References}

1) J. Takeya, M. Yamagishi, Y. Tominari, R. Hirahara, Y. Nakazawa, T. Nishikawa, T. Kawase, T. Shimoda, S. Ogawa, Appl. Phys. Lett., 90, 1021120 (2007).

2) S. Haas, Y. Takahashi, K. Takimiya, T. Hasegawa, Appl. Phys. Lett., 95, 022111 (2009).

3) H. Minemawari, T. Yamada, H. Matsui, J. Tsutsumi, S. Haas, R. Chiba, R. Kumai, T. Hasegawa, Nature. 475, 364 (2011).

4) R.W.I. de Boer, M.E. Gershenson, A.F. Morpurgo, V. Podzorov, Phys. Stat. Sol. A, 201, $1302(2004)$.

5) S.-S. Sun, N.S. Sariciftci, Organic Photovoltaics, Mechanisms, Materials and Devices, CRC Press, New York, 2005, p. 239 chap. 10.

6) H. Spanggaard, F.C. Krebs, Sol. Energy Mater. Sol. Cells., 83, 125 (2004).

7) C.W. Tang, Appl. Phys. Lett., 48, 183 (1986).

8) M. Hiramoto, H. Fujiwara, M. Yokoyama, Appl. Phys. Lett., 58, 10622 (1991).

9) M. Kikuchi, K. Takagi, H. Naito, M. Hiramoto, Org. Electron., 41, 118 (2017).

10) M. Kikuchi, S. Makmuang, S. Izawa, K. Wongravee, M. Hiramoto, Org. Electron., 64, 92 (2019).

11) M. Kikuchi, M. Hirota, T. Kunawong, Y. Shinmura, M. Abe, Y. Sadamitsu, A. M. Moh, S. Izawa, M. Izaki, H. Naito, M. Hiramoto, ACS Appl. Energy Mater., 2, 2087 (2019).

12) Y.Yuan, G. Giri, A.L. Ayzner, A.P. Zoombelt, S.C.B. Mannsfeld, J. Chen, D. Norduland, M.F. Toney, J. Huang, Z. Bao, Nat. Commun., 5, 3005 (2014).

13) K. Takimiya, S. Shinamura, I. Osaka, E. Miyazaki, Adv. Mater., 23, 4347 (2011). 
14) R. J. Chesterfield, J. C. McKeen, C. R. Newman, P. C. Ewbank, D. A. S. Filho, J-L. Brédas, L. L. Miller, K. R. Mann, C. D. Frisbie, J. Phys. Chem. B, 108, 19281 (2004).

(15) A.K.K. Kyaw, D.H. Wang, V. Gupta, W.L. Leong, L. Ke, G.C. Bazan, A.J. Heeger, ACS Nano., 7, 4569 (2013).

16) N. Shintaku, M. Hiramoto, S. Izawa, Org. Electron., 55, 69 (2018).

17) T. Kirchartz, F. Deledalle, P.S. Tuladhar, J.R. Durrant, J. Nelson, J. Phys. Chem. Lett., 4, 2371 (2013).

18) L.J.A. Koster, V.D. Mihailetchi, R. Ramaker, P.W.M. Blom, Appl. Phys. Lett., 86, $1(2005)$.

19) J. Gorenflot, M.C. Heiber, A. Baumann, J. Lorrmann, M. Gunz, A. Kampgen, et al., J. Appl. Phys., 115, 144502 (2014).

20) T. Kirchartz, B.E. Pieters, J. Kirkpatrick, U. Rau, J. Nelson, Phys. Rev. B., 83 115209 (2011).

21) N.V Elumalai, A. Uddin, Energy Environ. Sci ., 9, 391, (2016).

22) M. Hiramoto, M. Kikuchi, S. Izawa, Adv. Mater., 30, 1801236 (2018)]. 


\section{Figure Captions}

Fig. 1 (a) Structure of a lateral junction cell. (b) Photograph of a lateral cell ( $L=18 \mathrm{~mm})$. (c) Energy diagram of C8-BTBT and PTCDI-C8.

Fig. 2 (a) $J_{\mathrm{sc}}-V$ characteristics of cells with $L=0.2$ (green curve), 0.7 (black curve), 2 (blue curve), and $18 \mathrm{~mm}$ (red curve). Dependence on $L$ of (b) $J_{\mathrm{sc}}$, (c) $V_{\mathrm{oc}}$, and (d) FF. Simulated solar light of $100 \mathrm{mWcm}^{-2}$ was irradiated.

Fig. 3 (a) $J_{\mathrm{sc}}-V$ characteristics of cells with $L=2 \mathrm{~mm}$ under irradiation at various intensities of simulated solar light from 0.1 to 10 sun. (b) Logarithmic plot between $J_{\mathrm{sc}}$ and light intensity. (c) Dependence on $L$ of the slopes obtained from the light intensity plots in Fig. 3(b). (d) Semi-logarithmic plot between $V_{\mathrm{oc}}$ and light intensity. (e) Dependence on $L$ of $n$ obtained from the slope of the line in Fig. 3(d).

Fig. 4 Schematics of the movable-mask experiments for holes (upper) and electrons (lower). Semi-logarithmic plots (middle) between $J_{\mathrm{sc}}$ and the distance of mask edge from the hole-collecting electrode (blue points, right sides) and from the electron-collecting electrode (red points, left sides) for cells with $L=4,6$, and $18 \mathrm{~mm}$.

Fig. 5 (a) Schematics of hole movement with (lower) and without (upper) movable mask. (b) Profiles of the portions of electrons (red curves) and holes (blue curves) that can be collected by the electron- and hole-collecting electrodes. Only the carriers in the green-shaded region appeared as the photocurrent. (c) Dependence of $J_{\mathrm{sc}}$ on $L$. The calculated and observed $J_{\mathrm{sc}}$ are shown by the blue and red curves, respectively. (d) Trap-assisted recombination between a trapped electron and a free hole. (e) 
Trap-assisted recombination between a trapped hole and a free electron. (f) Bimolecular recombination. 
Fig. 1

(a)

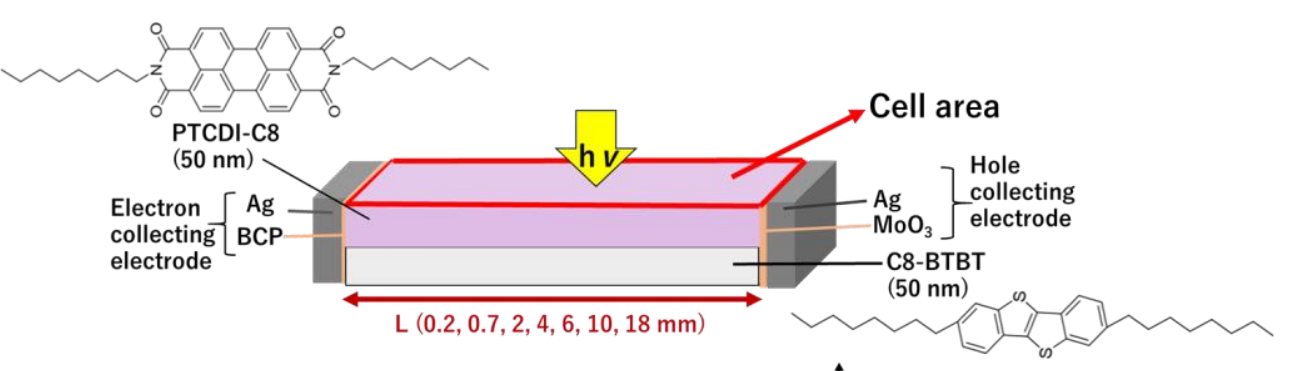

(b)

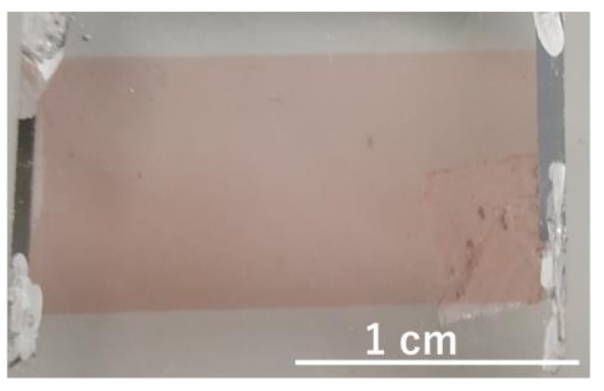


Fig. 2
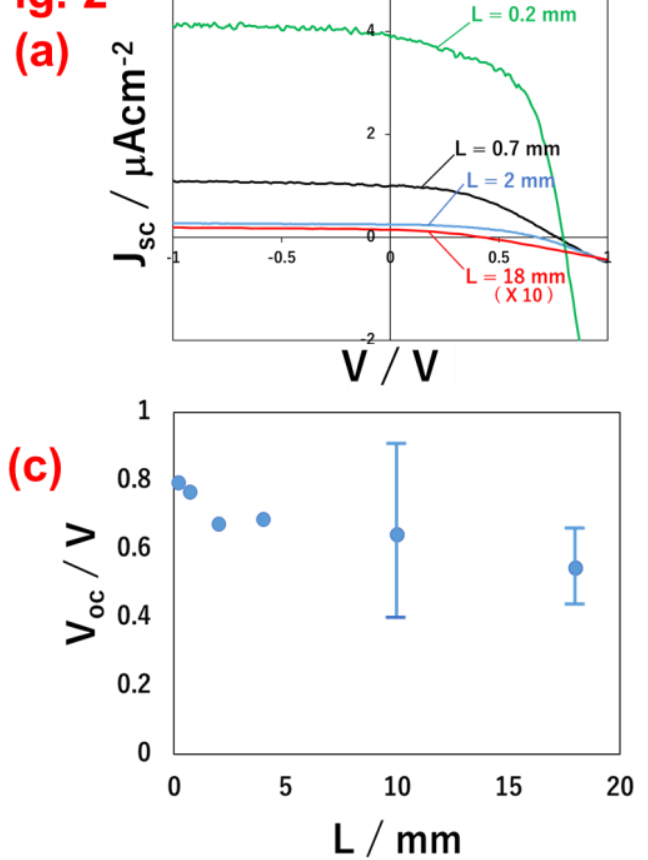

(b)

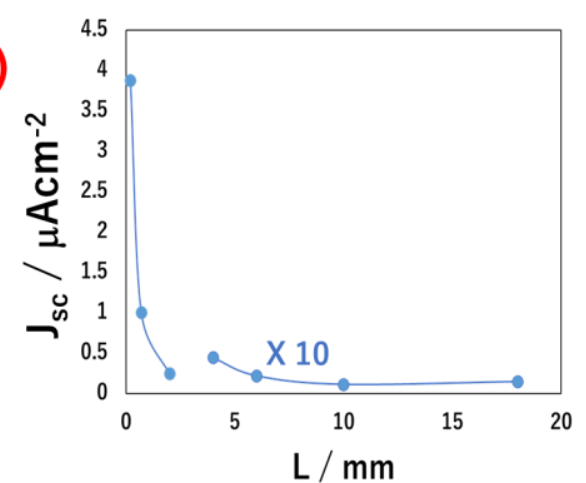

(d)

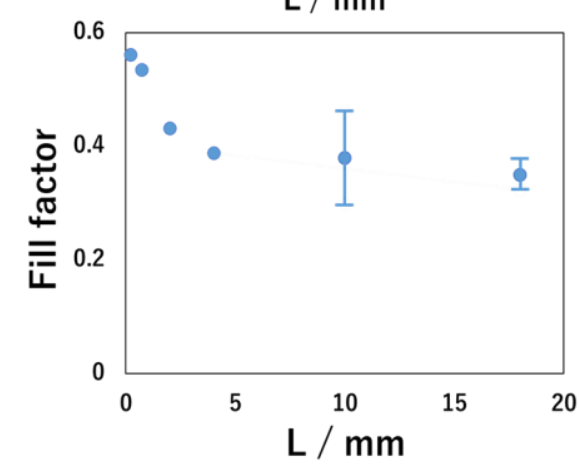


Fig. 3
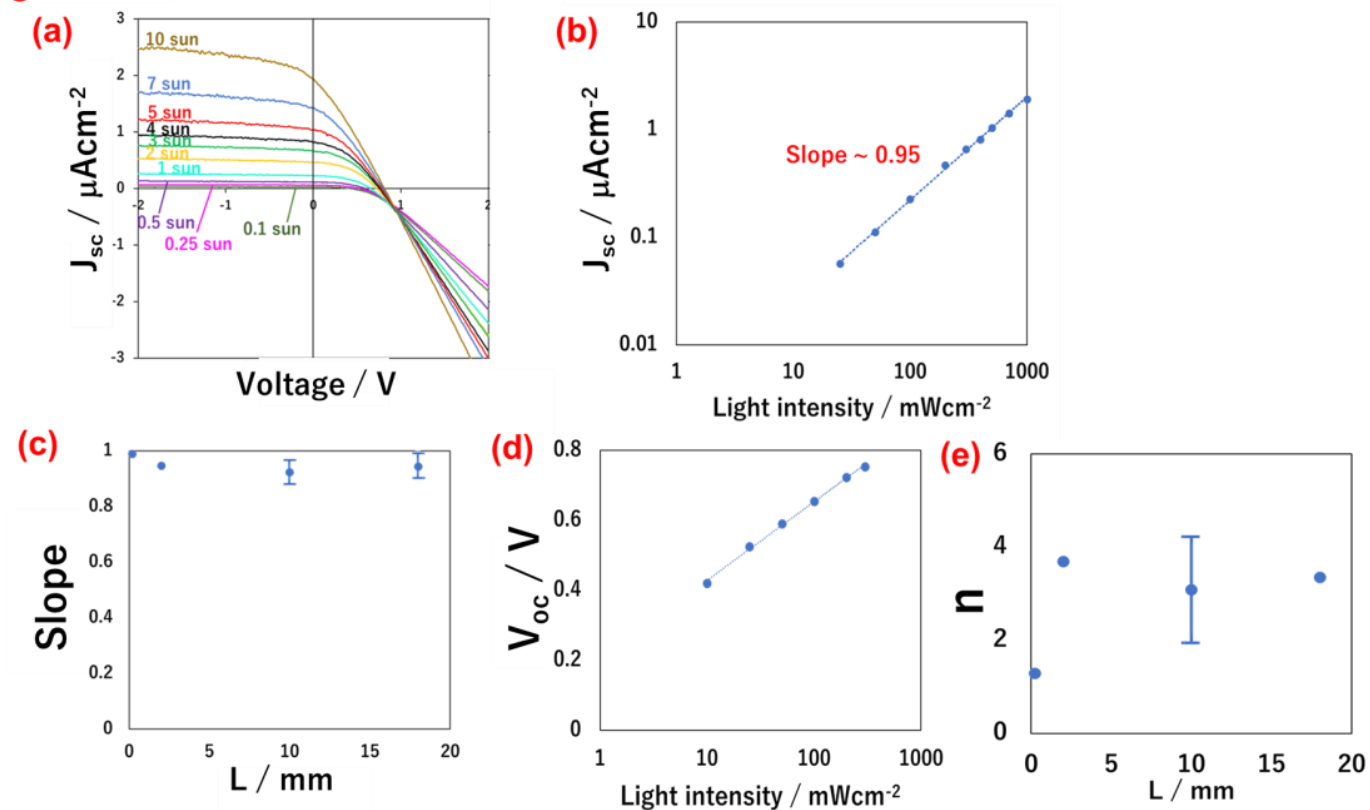
Fig. 4

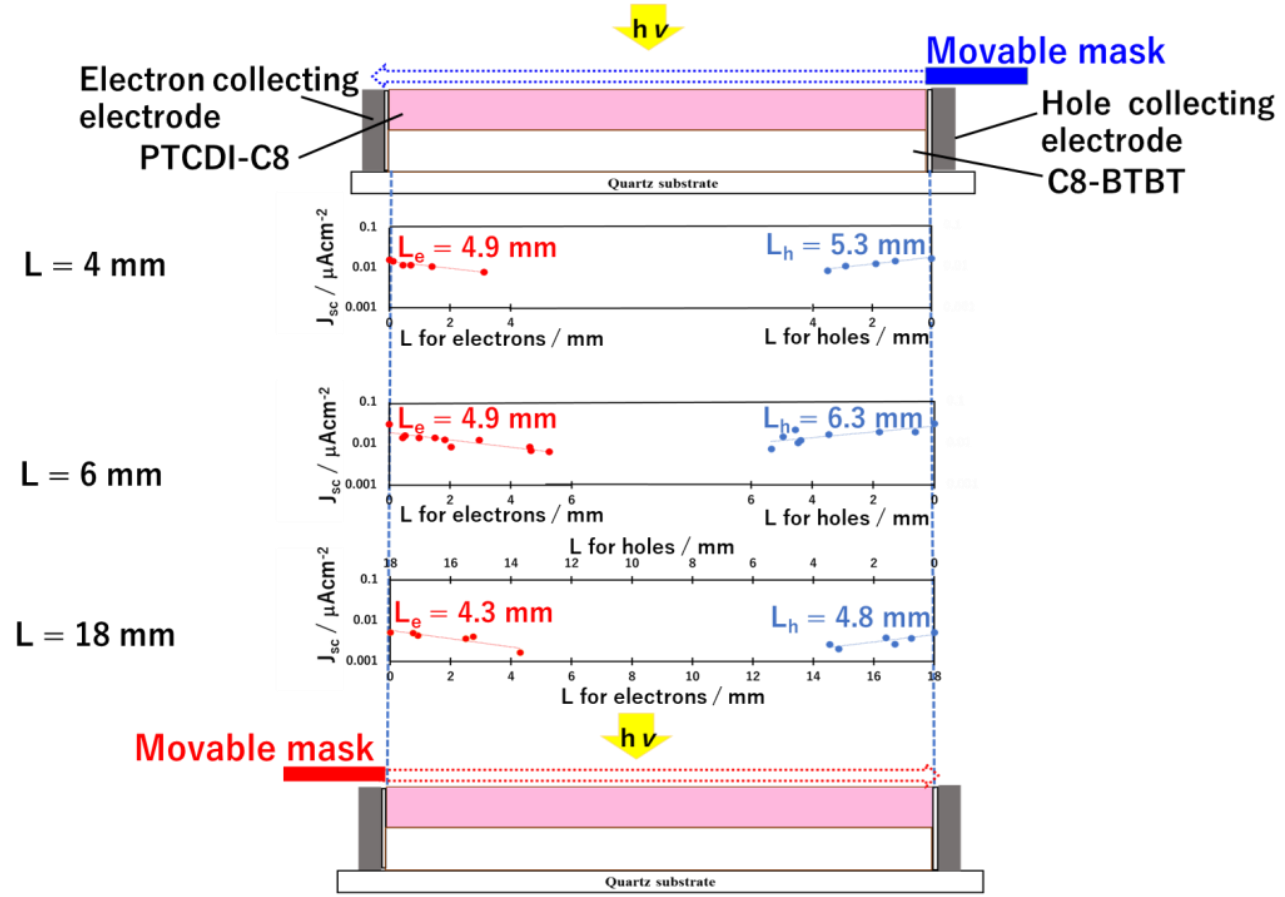


Fig . 5

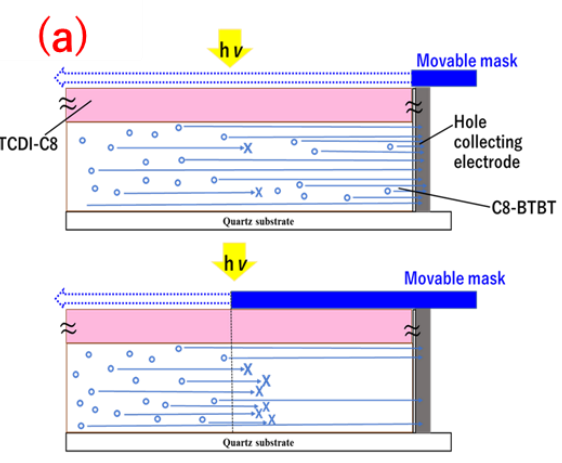

(b)

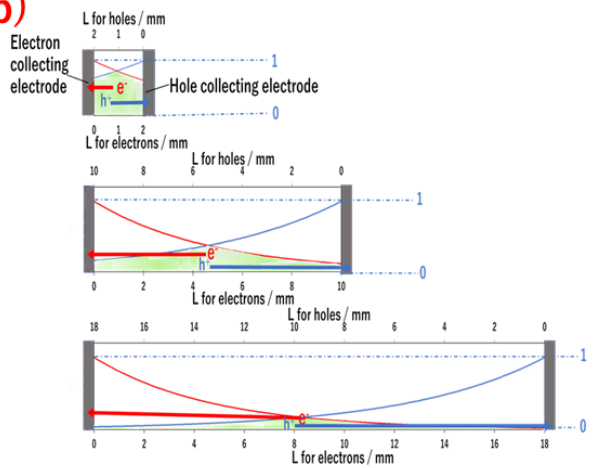

(c)

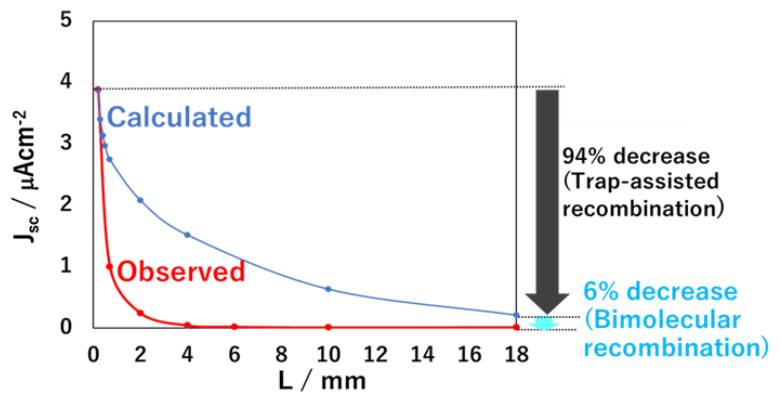

(d)

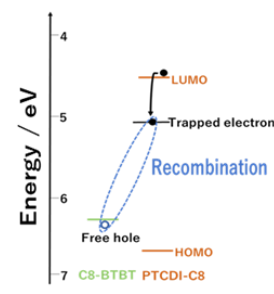

(e)

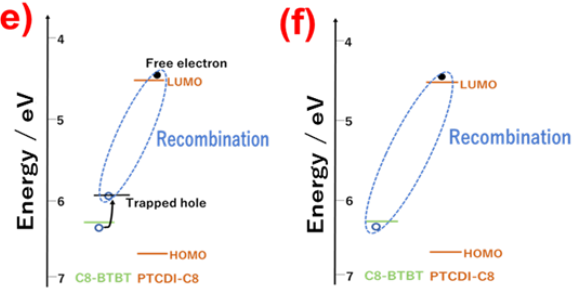


Fig. S1

(a)

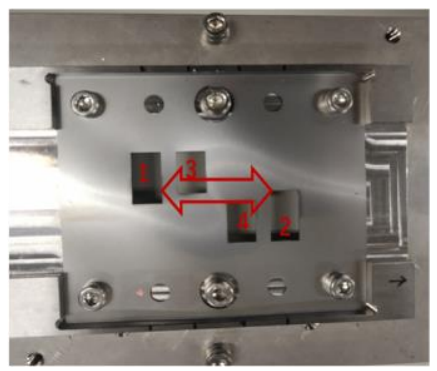

(b)

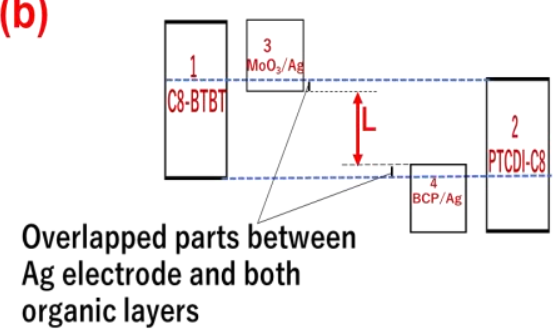

(c)

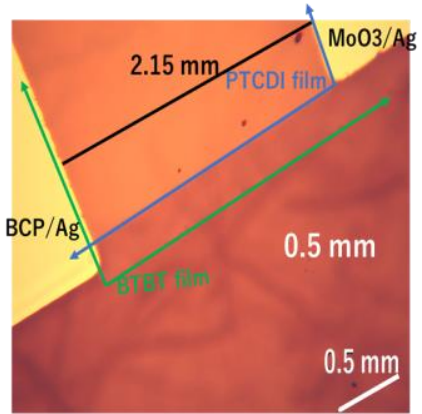

(d)

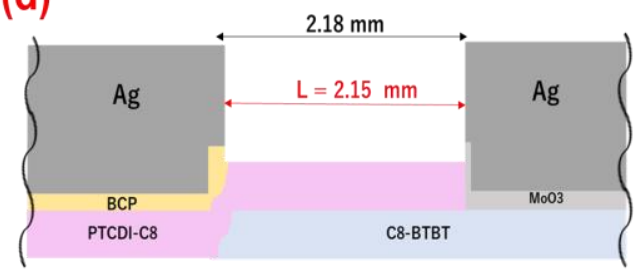

Fig. S1 (a) Photograph of a customized movable-mask system. (b) Design of the apertures in the mask. (c) Picture of one side of lateral cell $(L=2 \mathrm{~mm})$. (d) Detailed structure of lateral cell $(\mathrm{L}=2 \mathrm{~mm})$.

Using a precise positioning system, a quartz substrate set on the mask [Fig. S1(a)] was slid from side to side along the direction of the double arrow. C8-BTBT, PTCDI-C8, $\mathrm{MoO}_{3} / \mathrm{Ag}$, and BCP/Ag were deposited through the apertures in the order of aperture number from 1 to 4 .

The geometrical structures of cells, especially $L$ [Fig. S1(b)] and cell area, were precisely determined using optical microscopy. Fig S1 (c) shows picture of one side of lateral cell $(\mathrm{L}=2 \mathrm{~mm})$ taken by optical microscopy. Locations of BTBT film and PTCDI film are indicated by green and blue lines. By using this picture, electrode distance (L) was determined to $2.15 \mathrm{~mm}$. Overlapped distance between BTBT film and PTCDI film was determined to $2.18 \mathrm{~mm}$. Fig. S1 (d) shows the detailed structure of 
lateral cell in the real scale shown in Fig. S1 (c). Parts overlapped between the Ag electrode and both organic layers are determined to $30 \mu \mathrm{m}$ (left side) and $0 \mu \mathrm{m}$ (right side). Light was always irradiated from the sufficiently thick (100 nm) Ag electrode side to avoid photocurrent generation under these overlapped parts. 


\section{Fig. S2}

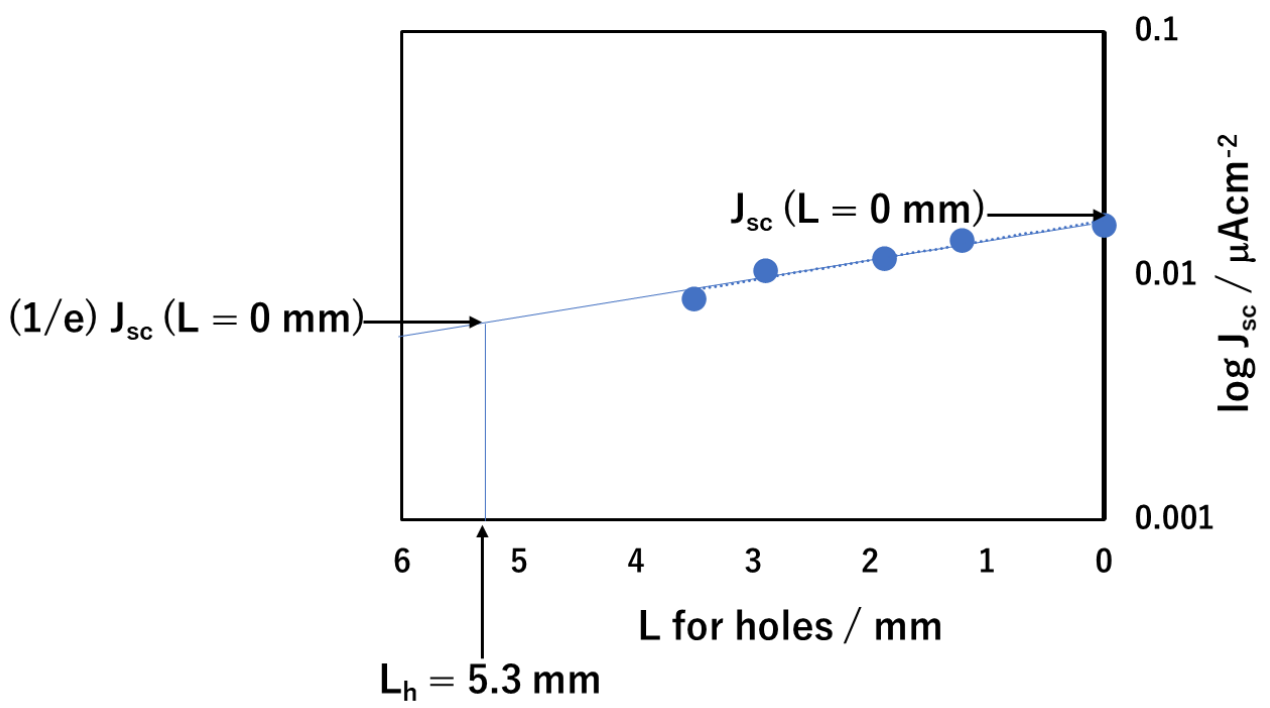

Fig.S2 Determination of hole diffusion length $\left(L_{\mathrm{h}}\right)$ for the cell having the electrode distance of $4 \mathrm{~mm}$.

Observed photocurrent den sity is $\mathbf{J}_{\mathrm{sc}}(\mathrm{L}=0 \mathrm{~mm})$ (full open of movable mask). Hole diffusion length $\left(L_{\mathrm{h}}\right)$ corresponds to the $\mathrm{L}$ value showing the photocurrent density of (1/e) $\mathrm{J}_{\mathrm{sc}}(\mathrm{L}=0 \mathrm{~mm})$. 